\title{
Complutum
}

ISSN: 1131-6993

\section{Una perspectiva desde la cotidianidad sobre la cultura material mursi (SW Etiopía)}

\author{
Juan Salazar Bonet ${ }^{1}$; Timothy Clarck²; Marcus Brittain ${ }^{3}$
}

Recibido: 4 de julio de 2016 / Aceptado: 6 de marzo de 2017

Resumen. Los mursi son un grupo agrícola-ganadero del sudoeste de Etiopía que construye unos poblados temporales denominados ôrri a bio. En estos asentamientos se emplea, de forma cotidiana, una serie de objetos realizados a partir de materiales locales abundantes mediante técnicas conocidas por la mayor parte de la población. Habitualmente, los estudios sobre identidades colectivas prestan menos atención a este tipo de objetos cotidianos y, en cambio, priorizan otros de carácter más extraordinario. Sin embargo, este conjunto de materiales, así como sus formas, funciones, significados y las prácticas asociadas a ellos, tienen un papel relevante a la hora de establecer quién pertenece a la comunidad y cómo lo hace. En el presente estudio se emplea el ôrri a bio como unidad de observación etnoarqueológica donde explorar la relación entre diversas materialidades y la construcción de un particular nosotros.

Palabras clave: Mursi; cultura material; agricultores-ganaderos; identidad.

\section{[en] A Perspective from Everyday Life on Mursi Material Culture (SW Ethiopia)}

Abstract. The Mursi are an agro-pastoralist group from south-west Ethiopia that builds temporary settlements called ôrri a bio. In these settlements, a series of objects that are made from local, abundant materials by using techniques known by most of the population, are used daily. Usually, studies on collective identities pay less attention to this type of everyday objects and prioritize others of a more extraordinary type. However, this set of materials, as well as their forms, functions, meanings and the practices associated with them, play a relevant role in establishing who and how belongs to the community. In the present study we use the ôrri a bio as an ethnoarchaeological unit of observation where we explore the relationship between diverse materialities and the construction of a particular us.

Keywords: Mursi; Material Culture; Agro-Pastoralist; Identity.

Sumario. 1. Introducción. 2. Los ôrri a bio mursi. 3. Una escala del mundo. 4. Conclusión.

Cómo citar: Salazar Bonet, J. et al.(2017): Una perspectiva desde la cotidianidad sobre la cultura material mursi (SW Etiopía). Complutum, 28(2): 431-443.

\section{Introdución}

La división del concepto de cultura en una serie de subcategorías, incluyendo la social, la religiosa, la económica y la material, ha condicionado desde sus inicios el estudio de aquello considerado tangible (Boas 1911; Schlanger 2012). Este particular planteamiento de la antropología se ha incorporado tanto al discur- so arqueológico como al etnográfico, favoreciendo a lo largo de las décadas la aparición de ámbitos de estudio sobre la materialidad en principio excluyentes, y a menudo opuestos. A modo de ejemplos se pueden mencionar el diferente tratamiento que empleó la primera etnografía para estudiar las fábricas y las artes, $\mathrm{o}$, ya en el siglo XX, la división entre aquello considerado profano y lo ritual, lo técnico y lo

\footnotetext{
Florida State University International Programs Valencia (España)

E-mail: jsalazarbonet@gmail.com

2 St Peter's College, University of Oxford (Reino Unido)

3 Cambridge Archaeological Unit, University of Cambridge (Reino Unido)
} 
simbólico o el estilo y la función. Esta aparente dicotomía también ha influido a la hora de explorar diversos materiales y objetos con los que las poblaciones se identifican y son identificadas.

Así, a la hora de analizar cómo se entienden y construyen a sí mismos los grupos humanos, tanto presentes como pasados, existe una tendencia a primar ciertos elementos sobre otros, que son considerados relevantes. Durante décadas, la arqueología, como ciencia de los objetos antiguos (Olsen et al. 2012), ha priorizado su interés en objetos de carácter foráneo, con un alto coste, de cierta complejidad tecnológica o decorativa, que son empleados en contextos singulares, mediante el uso de técnicas consideradas únicas o por representar la peculiaridad del individuo o del grupo frente a otros. Esta elección de objetos presenta una dilatada trayectoria histórica y ha aportado un conocimiento sin precedentes sobre los grupos del pasado. Sin embargo, la selección de determinados objetos enmascara una realidad cotidiana en la que personas, animales y medio interactúan de forma continua (Godelier 1986; Hodder 1986; Robb 1998; González-Ruibal 2006; Ingold 2007).

La etnoarqueología, como estrategia de la arqueología, ofrece la oportunidad de describir y analizar las prácticas cotidianas, que se revelan clave a la hora de construir y transmitir un modo particular de percibir y actuar en el mundo (Bourdieu 1977; Foucault 1988). La tecnología, la técnica y la cultura material son elementos vertebradores determinantes de toda comunidad, creando fructíferos ámbitos de estudio donde explorar las relaciones entre objetos y personas (González-Ruibal et al. 2010; Lemonnier 2012).

Los mursi son una de las poblaciones agrícola-ganaderas que habitan el curso bajo del río Omo, una región situada en el extremo sudoeste de Etiopía (Fig. 1). También en su caso se ha primado el estudio de una reducida tipología de objetos, los platos labiales (p. ej. dhebinya) que llevan las mujeres y las varas ( $\mathrm{p}$. ej. dongen) empleadas en los duelos masculinos, son considerados materializaciones de su identidad individual y colectiva (Turton 1979, 2002; LaTosky 2006; Fayers-Kerr 2011; Regi 2008). Los aproximadamente 10.000 mursi habitan $2.700 \mathrm{~km}^{2}$ en el margen izquierdo del río Omo, un área ocupada a lo largo de los últimos 200 años mediante diversos desplazamientos de población (Turton 1979) (Fig. 1).
Como muchos otros grupos del este africano para los que la ganadería juega un papel relevante, los mursi tienen sus principales instituciones de afiliación en el clan (kabicho), que establece vínculos consanguíneos entre individuos; las secciones locales (bhuran), que vertebran redes de cooperación en una escala territorial, y los grupos de edad (teri), que jerarquizan a la población en diferentes grados a lo largo de sus vidas (Turton 1973). A pesar de considerarse a sí mismos ganaderos, dos terceras partes del alimento consumido provienen de la producción cerealista. La agricultura, sus útiles y productos pertenecen, en general, a la esfera femenina, mientras que la ganadería pertenece a la masculina. Aunque coordinadas, los dos sexos mantienen un alto grado de independencia en las actividades cotidianas. Los mursi cultivan una cosecha en las riberas del Omo durante la temporada seca y una segunda en una llanura de interior durante la temporada húmeda. Para posibilitar ambas cosechas $\mathrm{y}$ mantener el ganado en la llanura central realizan un movimiento trashumante anual de ida y vuelta al río.

El presente trabajo identifica y describe una serie de tipologías y cadenas operativas de la cultura material mursi con el objetivo de explorar la construcción de un particular nosotros en el sudoeste de Etiopía. Los elementos propuestos no pueden considerarse signos, marcadores o emblemas materiales, como los platos labiales, las escarificaciones o las varas. En vez de estos, se analizan aquellos empleados de forma cotidiana para obtener, transformar y consumir alimentos en los poblados construidos durante la temporada húmeda en la llanura próxima al río Omo. Estos asentamientos son el lugar y el momento del año donde la comunidad desarrolla una "vida plena" (Turton 1973), convirtiéndolos en una unidad de observación pertinente (Jover 2013).

La información aportada en el presente artículo se obtuvo a lo largo de cinco campañas de trabajo de campo, realizadas entre 2010 y 2014, en diferentes meses del año y con duraciones de entre dos y cinco semanas. Durante estas campañas se realizaron observaciones, inventarios, planimetrías y entrevistas en diversos poblados temporales, un trabajo facilitado por la labor de traductores mursi con experiencia en este ámbito de la investigación. El presente estudio se enmarca dentro del Mursiland Heritage Project que, desde 2009, evalúa el potencial del curso bajo del río Omo 


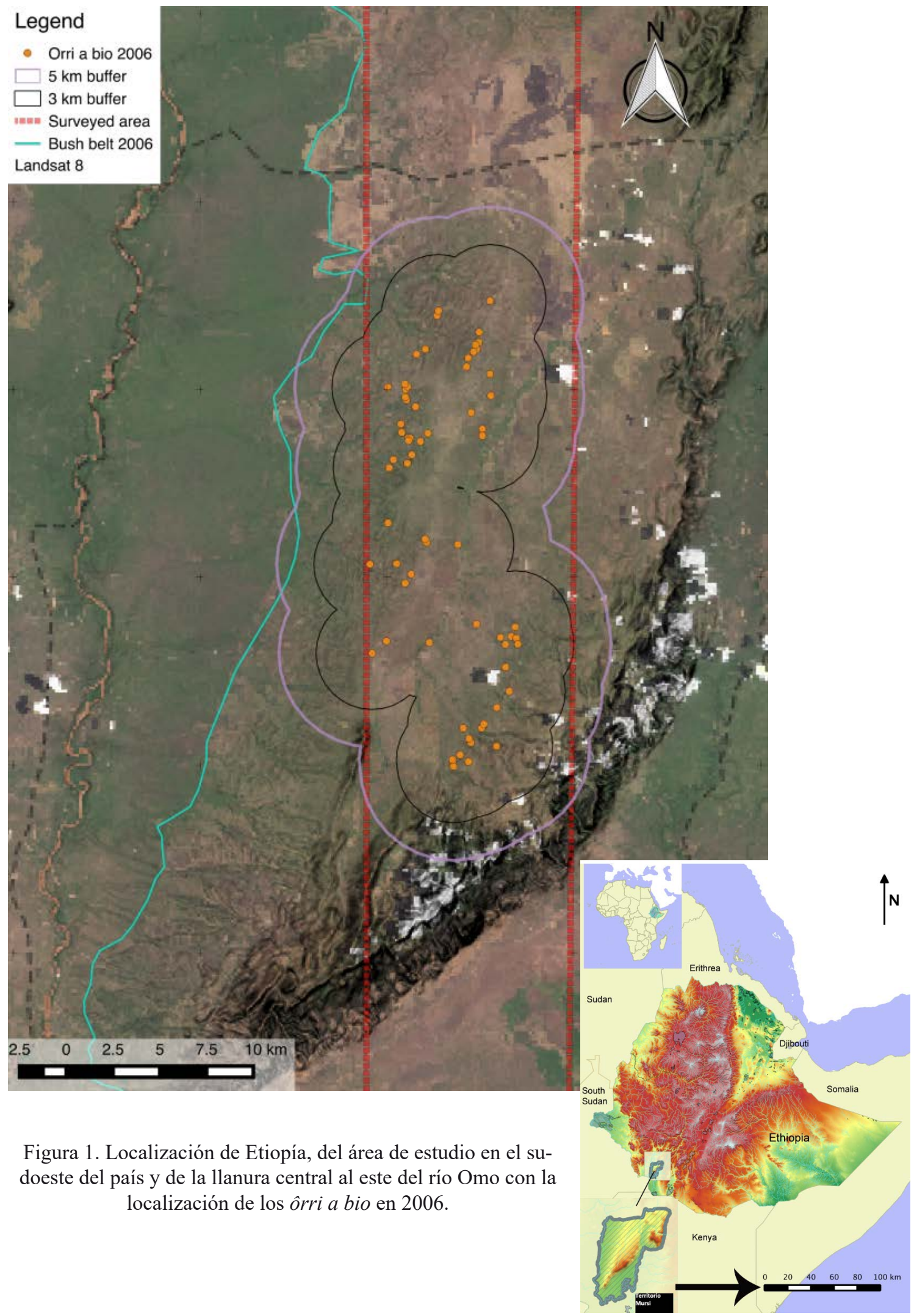


como espacio de estudio de las comunidades agrícola-ganaderas en la región a través de la arqueología y la etnohistoria (Clack y Brittain 2011a, 2011b) (http://mursi-archaeology. $\mathrm{com} /$ ). El proyecto ha evidenciado la dificultad de asociar materialidades y poblaciones en un contexto caracterizado por el cambio y la hibridación continua (Brittain et al. 2013; Bassi 2011; Epple 2014). De hecho, en los últimos cuatro años el ritmo de cambio se ha intensificado por un megaproyecto de transformación agrícola y el inicio de la sedentarización de las poblaciones trashumantes (Turton 2011).

\section{Los ôrri a bio mursi}

Los asentamientos agrícola-ganaderos mursi son construidos durante la temporada húmeda y habitados entre aproximadamente marzo y septiembre, no siempre en la misma localización. La población en uno de estos asentamientos temporales puede variar de una docena de personas a más de un centenar, aunque la disposición general del asentamiento a la hora de agruparse es similar. En ellos, uno o varios cercados circulares rodean un área de casas y corrales al aire libre en los que habitan hombres casados, sus esposas, la descendencia, ancianos y otros familiares y amigos. El conjunto de estos cercados con casas y corrales, sin tener en cuenta las diferencias de tamaño, se denomina ôrri a bio (Fig. 2).

Al construir un nuevo ôrri a bio, los hombres construirán un recinto perimetral de aproximadamente veinte metros de diámetro y una única entrada mediante la acumulación de matorrales espinosos. Mientras, la mujer o mujeres casadas construirán en su interior las viviendas, o dori. Éstas últimas trazan, una vez seleccionado el lugar donde se va a ubicar la casa, un círculo en la tierra utilizando una rama y empleando su cuerpo y brazo a modo de compás. Este círculo será el perímetro de la casa y en él se introducirán, cada 10 $\mathrm{cm}$ aproximadamente, ramas flexibles y verticales. El aspecto definitivo semiesférico de una vivienda se obtiene cuando se arquean y estabilizan estas ramas verticales a una altura de aproximadamente un metro y medio. Posteriormente, se consolida la estructura trenzando horizontalmente ramas flexibles y, finalmente, se coloca el techo de haces de herbáceas. Las viviendas disponen de dos hogares, uno en el interior y en posición central de la casa, y el se- gundo a pocos metros frente a la entrada de la misma, ambos compuestos por tres piedras con dimensiones similares. Las tres piedras utilizadas en los hogares recuerdan a los tres días de luto que se guarda tras la muerte de una mujer, considerándose esta cifra un número femenino recordado en otros contextos (Eczet 2012).

El interior de un dori se divide en dos espacios, uno masculino a la izquierda de la entrada y otro femenino e infantil a la derecha, cada uno de ellos con diversos objetos al alcance de la mano. La totalidad de los objetos femeninos que hacen posible la continuidad del hogar se conoce como ahakesuim. Esta palabra se asocia a un segundo término, korrei, empleado para denominar a los objetos que las mujeres cargan en la cabeza cuando se desplazan a un nuevo asentamiento. Por su parte, los elementos masculinos, ahahirim, consisten en las armas, terneros recién nacidos y otros objetos, como los reposacabezas o diversos contenedores tubulares, que son transportados por los hombres.

En el exterior del cercado y frente a su entrada, se selecciona un espacio abierto denominado bal, donde los maridos se reúnen para hablar, discutir y consumir los alimentos, habitualmente en la proximidad de la sombra de un árbol. Las mujeres se reúnen en el interior del recinto cercado, en la proximidad de la casa, también al aire libre y bajo la sombra de arbusto. Este espacio es el lugar habitual de trabajo, diálogo y discusión femenino, donde se localiza, además, una área de actividad vinculada directamente a la transformación y la preparación del cereal.

La molienda es, por el tiempo empleado, una de las principales actividades femeninas diarias. El molino es propiedad de la mujer casada, que en ocasiones puede compartir su uso con otras mujeres de la familia. Los molinos de vaivén que emplean tienen una planta ovoide alargada, un perfil cóncavo en su eje transversal y rectangular en el longitudinal (Fig. 3). El grosor de estos útiles presenta una alta variabilidad, consecuencia del diferente grado de abrasión que haya sufrido la superficie activa o de trabajo, tanto por su uso diario como por el continúo repiqueteado para que no pierdan su rugosidad. Los molinos se elaboran con percutores de basalto a partir de bloques de granito recogidos en un radio de varios kilómetros, ya sea en forma de bloques desplazados a causa de arroyadas o desprendiendo piezas de relieves escarpados. 


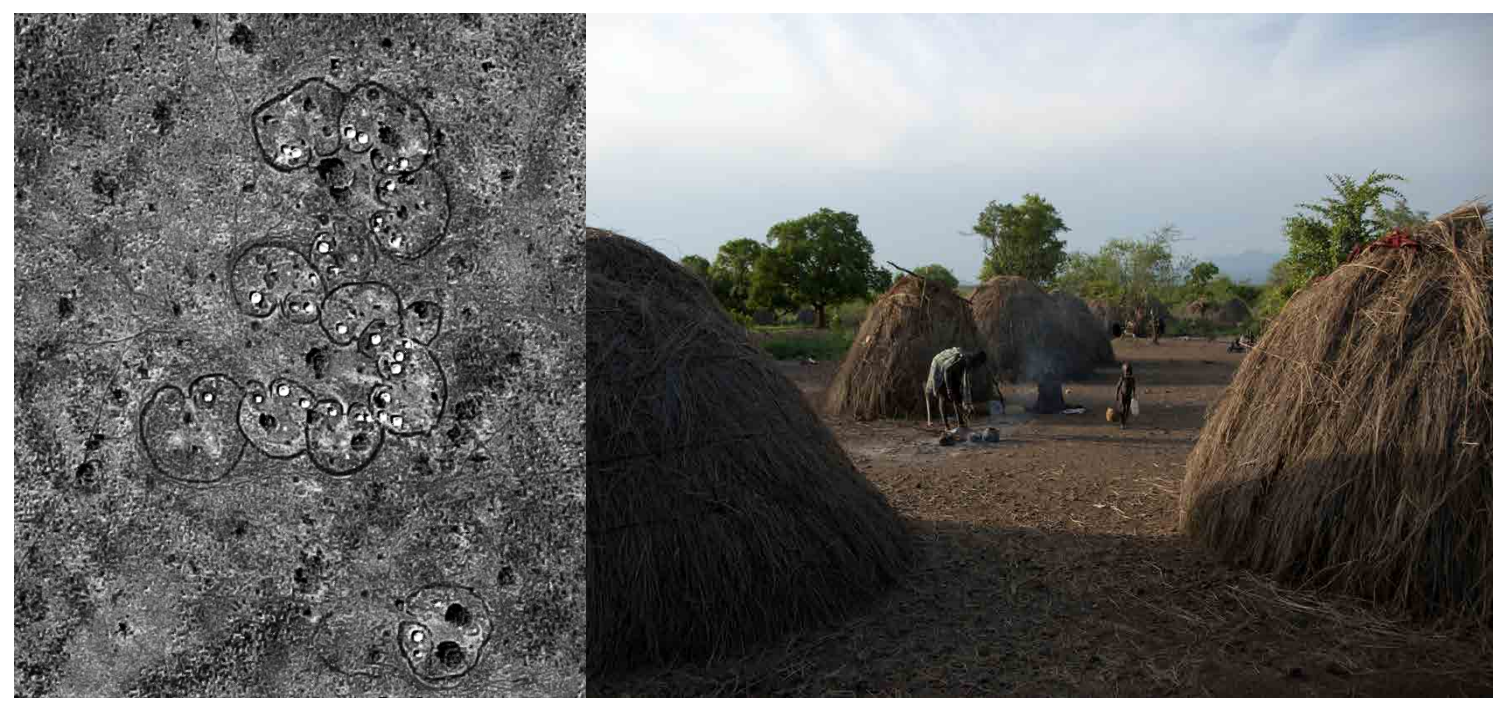

Figura 2. La palabra $\hat{o} r$ se traduce como vivienda, casa u hogar y, en plural, como casas o poblado. En segundo lugar, la palabra bio, es el plural de vaca, pero también el genérico para designar al ganado bovino. La imagen izquierda muestra una vista aérea de un asentamiento en la llanura mientras que la imagen de la derecha el interior de uno de estos poblados.

Cuando la mujer comienza el trabajo de molienda se coloca de rodillas junto a un lado corto del molino, elevándolo mediante la colocación de una o dos piedras bajo la superficie del extremo próximo a ella. El esfuerzo muscular que realiza la persona que muele se ve facilitado por la inclinación en varios grados que otorgan estas piedras. Otro factor que hace más sencillo el movimiento de vaivén es la ligera curvatura ascendente de la superficie activa del molino en su extremo distal, que desacelera el movimiento de la moledera y minimiza el esfuerzo físico de la persona (Fig. 4). Una serie de canciones ayudan a mantener el ritmo y la respiración en el la molienda, así como el aprendizaje de los movimientos en las niñas y las adolescentes (Fig. 5).

Las mujeres también emplean a diario los vasos cerámicos para cocinar en los hogares, tanto en aquellos en el interior de la vivienda como los ubicados en el exterior. Se elaboran y distinguen dos tipos de vasos, denominados ju, pl. junya, y dôle, pl. dolya. Ambos tipos corresponden a recipientes globulares en los que los cuellos presentan un ángulo más o menos pronunciado respecto del cuerpo, bordes salientes, labios planos, asas de sección elíptica y bases convexas. A pesar de las similitudes morfológicas entre estos dos tipos de vasos, sus dimensiones permiten clasificarlos en dos categorías distintas (Fig. 6). Cada mujer casada tiene en propiedad sus vasos aunque no

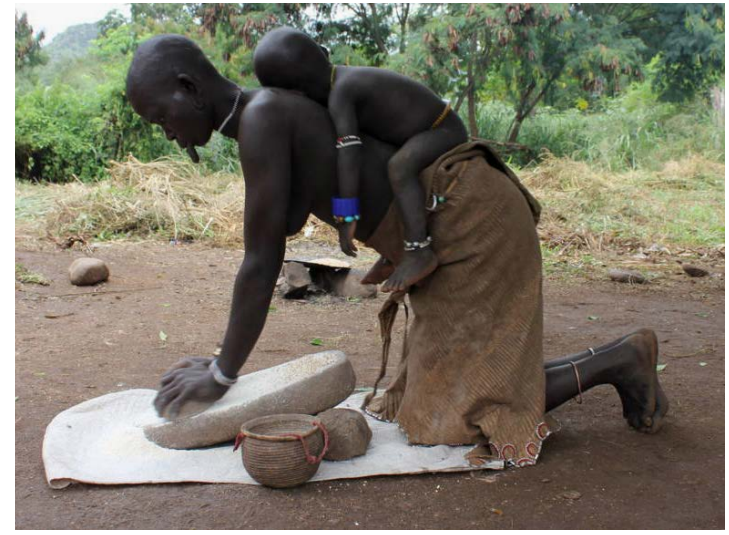

Figura 3. Las palabras molino y moledera hacen referencia, mediante una metáfora, a las palabras madre y bebé. Una moledera debe acoplarse en un molino como un recién nacido en la espalda de su madre (Foto J. Robitaille).

todas las mujeres los elaboran; con frecuencia se recurre a familiares y amistades con especial destreza para fabricarlos. Los principales pasos en el análisis de la cadena operativa reflejan los objetivos de cada fase, los materiales empleados, las acciones llevadas a cabo y su duración (Tabla 1).

Las técnicas decorativas aplicadas a los vasos mursi tienen una distribución geográfica homogénea en la llanura, se repiten de forma estandarizada y consisten en decoraciones 


\begin{tabular}{|c|c|c|c|}
\hline Pasos/fases & Objetivo & Materiales & Acción \\
\hline 1 & $\begin{array}{l}\text { Obtener forma có- } \\
\text { nica, base del futuro } \\
\text { vaso }\end{array}$ & $\begin{array}{c}\text { Fragmento de arcilla } \\
\text { del tamaño aproximado } \\
\text { de un puño humidifica- } \\
\text { do con agua. }\end{array}$ & $\begin{array}{l}\text { La ceramista amasa el fragmento de arcilla. } \\
\text { Cuanto este obtiene la plasticidad adecuada } \\
\text { inicia su moldeado mediante golpes repetidos y } \\
\text { continuados sobre la rodilla, a modo de molde. }\end{array}$ \\
\hline 2 & $\begin{array}{l}\text { Iniciar la elaboración } \\
\text { de las paredes del } \\
\text { vaso por la adición } \\
\text { de sucesivos colom- } \\
\text { binos en un plano } \\
\text { vertical }\end{array}$ & $\begin{array}{l}\text { Arcilla con forma } \\
\text { cónica alargada } \\
\text { Agua para humidificar } \\
\text { Base de gramíneas } \\
\text { trenzadas para soportar } \\
\text { el vaso sin que este se } \\
\text { desplace. }\end{array}$ & $\begin{array}{c}\text { La ceramista trabaja el fragmento de arcilla des- } \\
\text { plazándolo entre sus dos manos hasta obtener un } \\
\text { primer colombino. Este se moldea a lo largo del } \\
\text { borde de la forma cónica anterior. }\end{array}$ \\
\hline 3 & $\begin{array}{l}\text { Alisar las paredes } \\
\text { exteriores e interio- } \\
\text { res del vaso }\end{array}$ & $\begin{array}{l}\text { Fragmento de calabaza } \\
\text { y la acción humificado- } \\
\text { ra del agua. }\end{array}$ & $\begin{array}{l}\text { La ceramista regulariza las superficies resultado } \\
\text { de la unión de ese primer colombino y la forma } \\
\text { inicial cónica. Usa sus dedos pasándolos por la } \\
\text { junta entre ambos y posteriormente los regulari- } \\
\text { za pasando por las superficies un fragmento de } \\
\text { calabaza humificado. }\end{array}$ \\
\hline 4 & $\begin{array}{l}\text { Obtener el tamaño } \\
\text { deseado para el cuer- } \\
\text { po de la cerámica }\end{array}$ & $\begin{array}{l}\text { Arcilla, agua y frag- } \\
\text { mento de calabaza. }\end{array}$ & $\begin{array}{l}\text { La ceramista repite sucesivamente los pasos de } \\
\text { la acción anteriores ( } 2 \text { y } 3 \text { ). Elaboración de un } \\
\text { colombino, su aplique a la pared del vaso y su } \\
\text { regularización mediante la acción moldeadora } \\
\text { de los dedos y el uso del agua y del fragmento } \\
\text { de calabaza. }\end{array}$ \\
\hline 5 & $\begin{array}{l}\text { Elaboración del } \\
\text { borde el vaso }\end{array}$ & $\begin{array}{l}\text { Arcilla, agua y frag- } \\
\text { mento calabaza. }\end{array}$ & $\begin{array}{c}\text { Elaboración de un colombino de menor tamaño } \\
\text { que pasa a aplicarse a la forma globular obteni- } \\
\text { da previamente. Se repite en paso } 3 \text {. }\end{array}$ \\
\hline 6 & $\begin{array}{l}\text { Decoración impresa } \\
\text { del vaso }\end{array}$ & $\begin{array}{l}\text { Arcilla, agua y cordel } \\
\text { de dos fibras vegetales } \\
\text { trenzadas. }\end{array}$ & $\begin{array}{l}\text { Aplicación de un cordel trenzado por diversos } \\
\text { espacios de la superficie exterior del vaso. }\end{array}$ \\
\hline 7 & $\begin{array}{l}\text { Elaboración y apli- } \\
\text { que de los elementos } \\
\text { de prensión simé- } \\
\text { tricos }\end{array}$ & $\begin{array}{l}\text { Arcilla, agua, fragmen- } \\
\text { to de calabaza y tallo } \\
\text { de } 10 \mathrm{~cm} \text {. }\end{array}$ & $\begin{array}{l}\text { La ceramista moldea dos fragmentos de arcilla } \\
\text { de similares dimensiones y formas. Aplica el } \\
\text { primero de ellos en un lateral del vaso y coloca } \\
\text { un tallo sobre la boca del vaso para ubicar el } \\
\text { segundo. Regulariza con la acción de los dedos, } \\
\text { el agua y el fragmento de calabaza. }\end{array}$ \\
\hline 8 & $\begin{array}{l}\text { Decoración con } \\
\text { cordón e impresa } \\
\text { del vaso }\end{array}$ & $\begin{array}{l}\text { Arcilla, agua y cordel } \\
\text { de dos fibras vegetales } \\
\text { trenzadas. }\end{array}$ & $\begin{array}{l}\text { La ceramista pellizca la superficie alrededor de } \\
\text { la parte central del vaso, creando un cordón de } \\
\text { apenas un cm de grosor. A este cordón se le apli- } \\
\text { ca por su superficie la decoración mediente un } \\
\text { cordel trenzado. Aplique de botones de arcilla } \\
\text { circulares en el cordón y las asas. }\end{array}$ \\
\hline \multicolumn{4}{|l|}{$\begin{array}{l}\text { Secado de la } \\
\text { cerámica }\end{array}$} \\
\hline 9 & $\begin{array}{l}\text { Bruñido de las su- } \\
\text { perficies del vaso }\end{array}$ & Brazalete de hierro. & $\begin{array}{c}\text { La ceramista pasa por las superficies lisas sin } \\
\text { decoración un brazalete de hierro, en ocasiones } \\
\text { esta acción también se puede llevar a cabo con } \\
\text { una piedra. }\end{array}$ \\
\hline 10 & Acabado de la forma & Objeto cortante. & $\begin{array}{l}\text { La ceramista elimina posibles imperfecciones } \\
\text { de la forma. } \\
\text { Este paso no siempre es necesario. }\end{array}$ \\
\hline $\begin{array}{c}11 \\
\text { No observado } \\
\text { en Makki por } \\
\text { razones me- } \\
\text { teorológicas }\end{array}$ & $\begin{array}{l}\text { Cocción de la } \\
\text { cerámica }\end{array}$ & $\begin{array}{l}\text { Estructura excavada en } \\
\text { la tierra, leña y fuego. }\end{array}$ & $\begin{array}{l}\text { La ceramista excava un hoyo con las dimensio- } \\
\text { nes necesarias para ubicar los vasos y coloca } \\
\text { leña en la base del hoyo. Se cubre la cerámica } \\
\text { con leña y se le prende fuego a la hoguera. }\end{array}$ \\
\hline
\end{tabular}
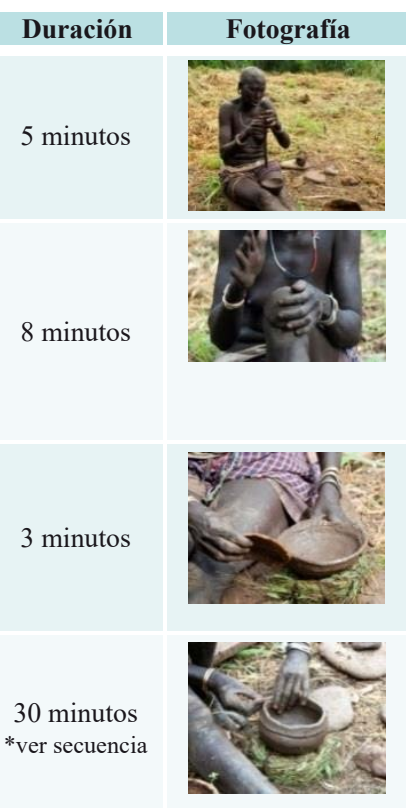

8 minutos

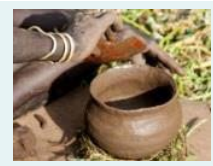

5 minutos

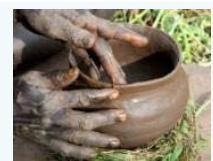

6 minutos
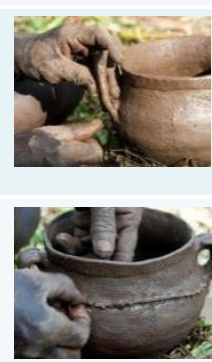

aprox.

20 horas

15 minutos

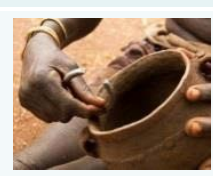

40 minutos

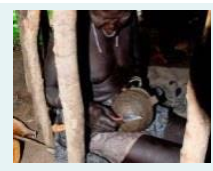

Varias horas

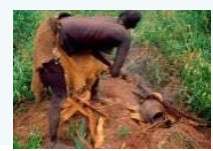

Tabla 1. El urdido es empleado para elaborar ambos tipos de vasos. Éstos se realizan preferentemente durante la temporada seca y en las riberas del río Omo, donde existen abundantes depósitos de arcilla. 


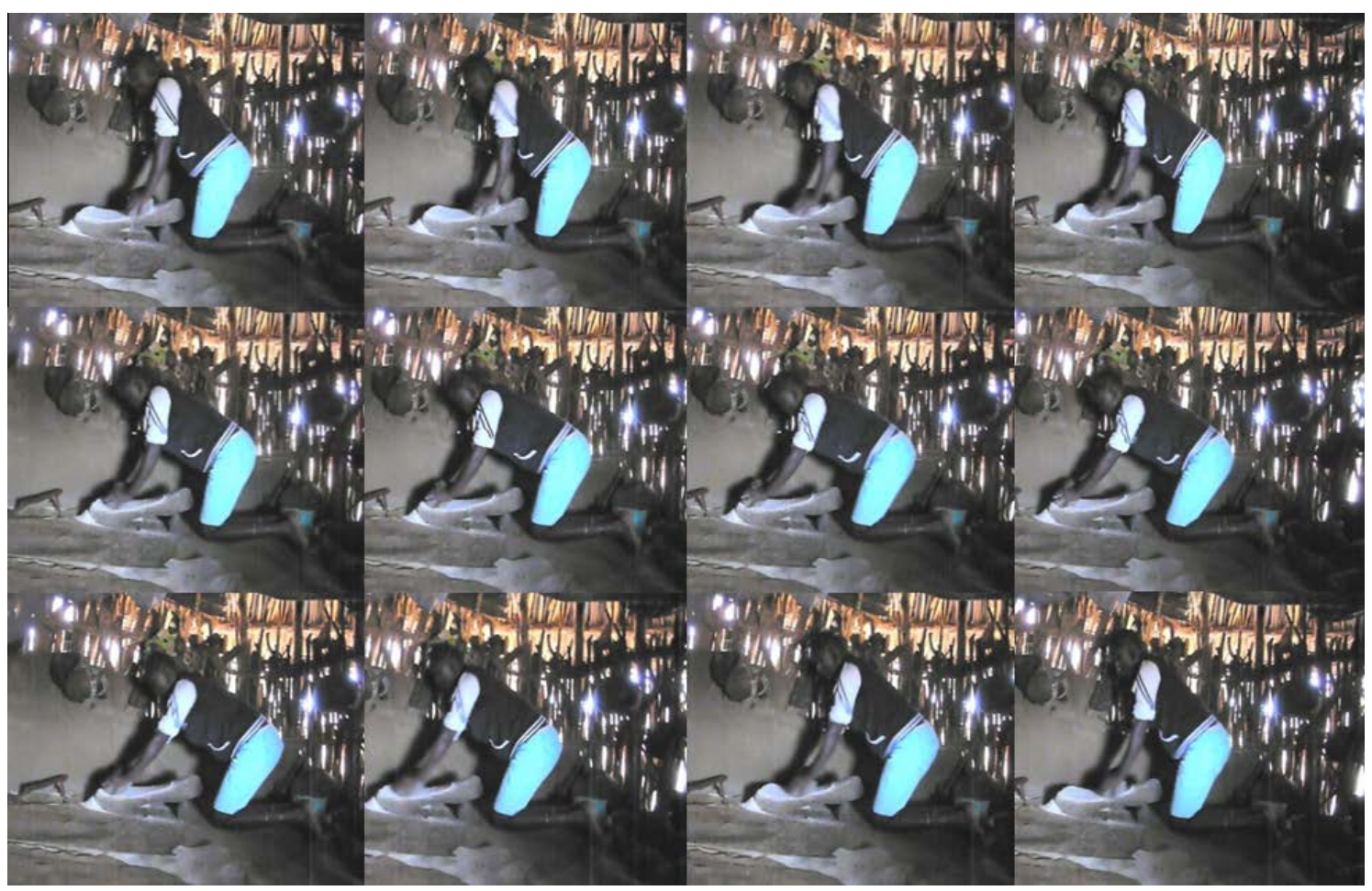

Figura 4. Secuencia en imágenes del recorrido de molienda durante cuarenta décimas de segundo.
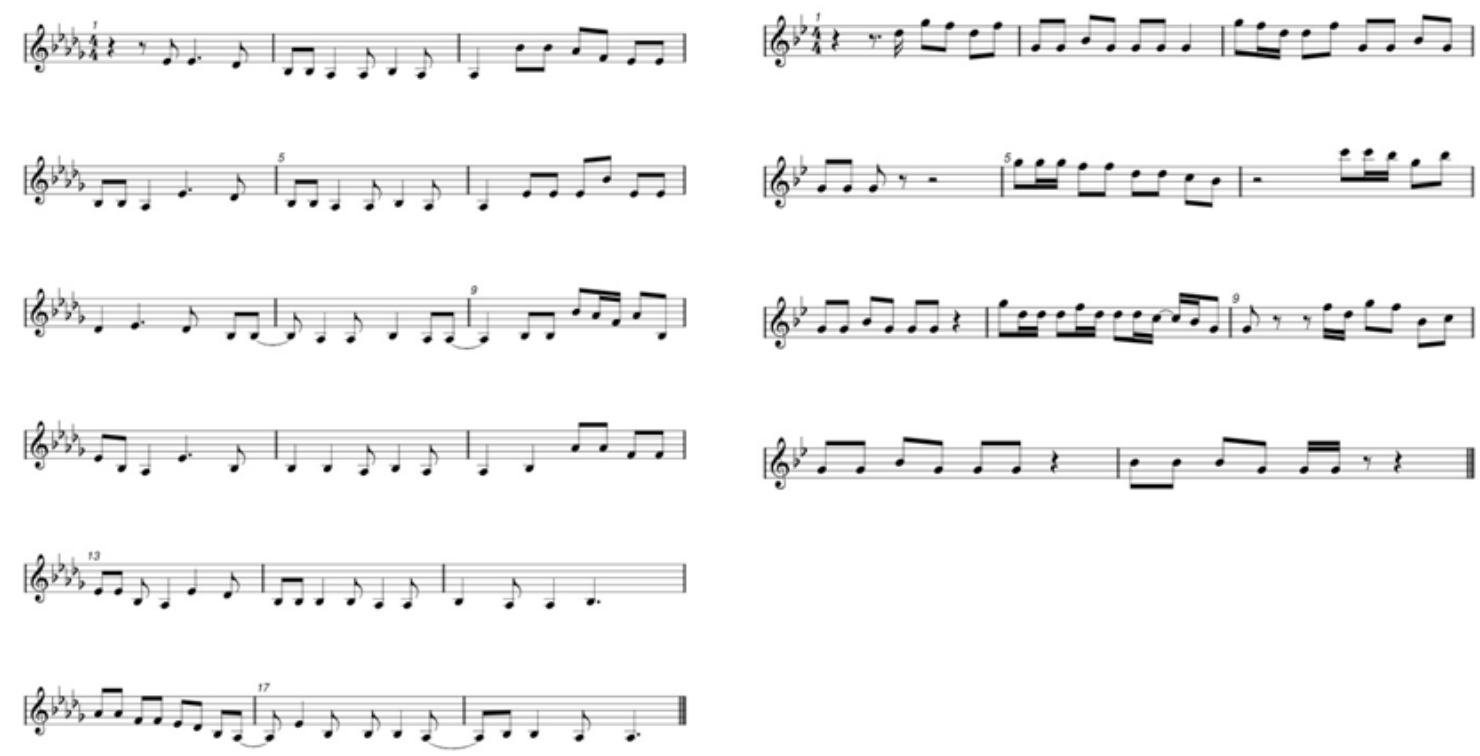

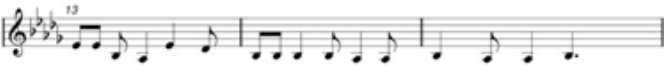

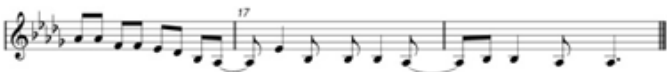

Figura 5. Partituras de dos canciones mursi de moler. El compás corresponde a un doble recorrido de la moledera a lo largo del molino, facilitando la respiración durante la acción. 

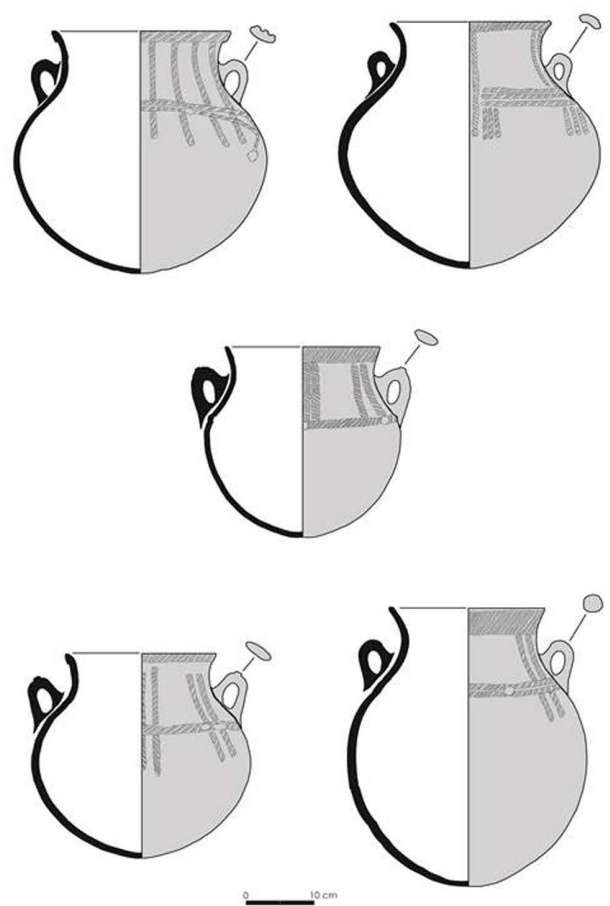
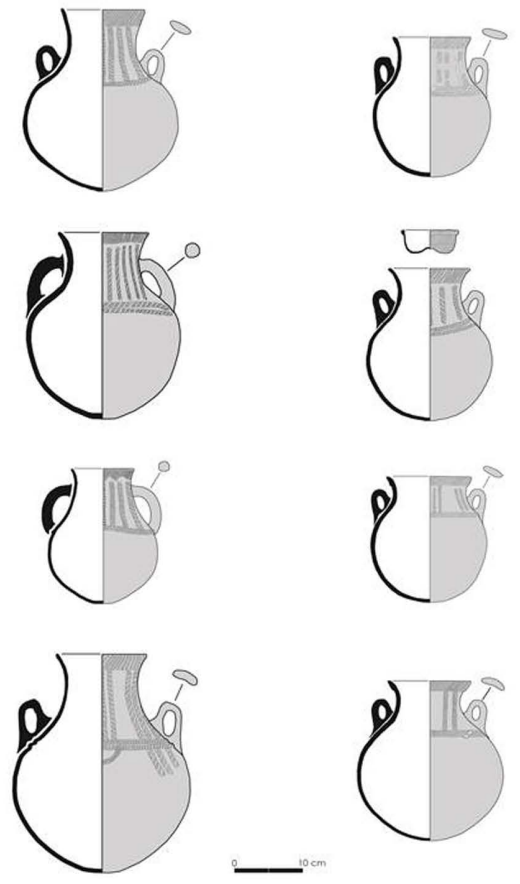

Figura 6. Tipología de vasos cerámicos, junya en la parte izquierda y dole en la parte derecha. Las formas de ambos vasos son cerradas o muy cerradas, además de profundas.
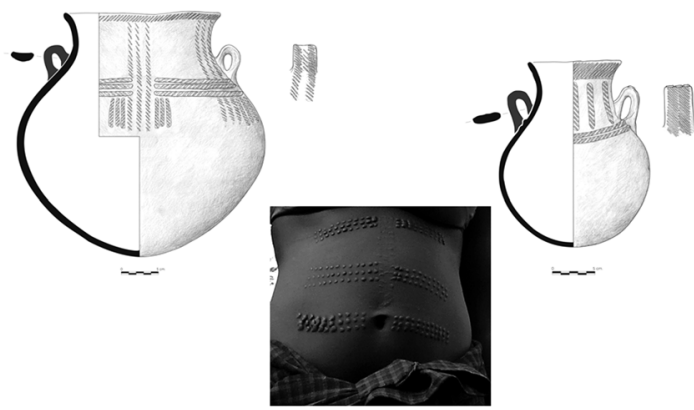

Figura 7. Los temas decorativos en los vasos se desarrollan en tres espacios, en labios y asas y en un friso en la mitad superior del vaso con un recorrido horizontal.

impresas con cuerda, cordones rectilíneos impresos con esta misma técnica, mamelones y botones. La distribución mixta de los motivos seriados compone tramas o patrones verticales y horizontales. En la parte central de los vasos las composiciones muestran una decoración vertical de cintas interrumpida por decoraciones horizontales, también longitudinales, impresas sobre cordón. Tanto la posición como los patrones decorativos recuerdan a tiras de cuero o fibras vegetales trenzadas que se utilizan para transportar otros contenedores realizados en madera o calabazas. Un tipo de escarificación femenina realizada en el vientre con motivo de la madurez sexual y la fecundidad, compuesto por motivos de bandas horizontales y verticales, muestra similitudes con los empleados en la decoración cerámica (Fig. 7).

Tres elementos morfológicos presentan indicios sobre la funcionalidad de los vasos, como son su forma globular, óptima para el transporte, sus bases convexas para ubicarlos sobre hogares y su boca estrecha para evitar la excesiva evaporación de los alimentos hervidos (Prussin 1987; Grillo 2014). El modo habitual de consumir el cereal es mediante la cocción de gachas en los junya. Los junya también se emplean para transportar agua y elaborar cerveza, café y aguamiel; ocasionalmente, se utilizan para hervir carne, para cocinar sopas y para remojar el grano de cereal con el fin de que sea más blando antes de la 


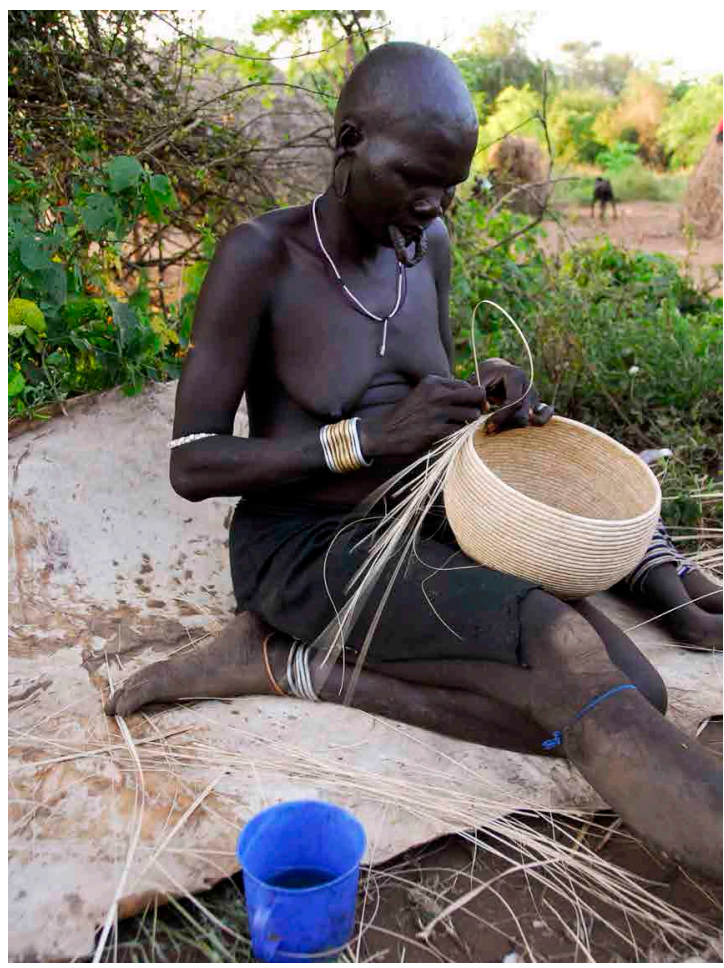

Figura 8. En la elaboración de una cesta se entrelazan, con un punzón, haces horizontales y verticales hasta obtener la forma deseada.

molienda. Por otra parte, los dolya se utilizan fundamentalmente para la cocción de un tipo de hojas silvestres llamadas kinoi, con las que se acompañan las gachas.

Las gachas se sirven y consumen en unas cestas trenzadas de fibras vegetales denominadas garchu. Éstas cestas son globulares y de boca abierta, sin cuello, con bordes salientes de sección ovalada y los labios y las bases planas. Para elaborar las cestas las mujeres cortan las hojas de una palmera local (Hyphaene thebaica) y rompen, separan y humedecen las fibras. La cestería mursi combina el trenzado de fibras horizontales, unidas verticalmente entre sí por medio de fibras planas. La forma inicial comienza por la base, mediante el tejido de una primera espiral, a la cual se fijan nuevos segmentos que, ampliándose y modificando el ángulo respecto de la base, permite construir las paredes de la cesta (Fig. 8). Se elaboran dos tipos de cestas que, al igual que en los vasos cerámicos, se distinguen por su tamaño. La principal función de las cestas de mayor tamaño es servir y consumir las gachas y transportar el grano durante la siembra. La cesta garchu es un regalo de esponsales de la
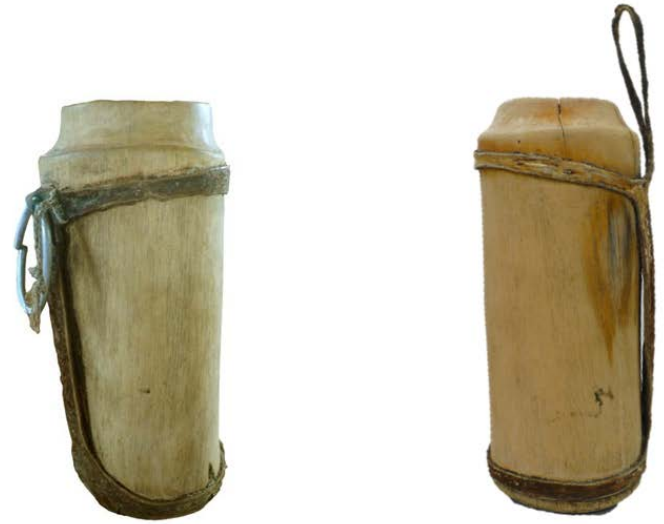

Figura 9. Los bagais documentados reflejan una alta homogeneidad en cuanto a tamaño y capacidad. En la actualidad y para su función de transporte comienzan a ser sustituidos por botellas de plástico de litro y medio.

novia a su futuro marido, y en ella le servirá los alimentos cada día. Las adolescentes emplean las cestas de menor tamaño para recolectar bayas o transportar pequeñas cantidades de grano.

El cereal obtenido y transformado por las mujeres no es suficiente para garantizar la supervivencia de las distintas personas que componen un ôrri a bio, en especial durante determinados periodos del año y para determinadas franjas de edad de sus miembros. Por ello, los productos derivados de la ganadería son un complemento imprescindible de la dieta cerealista. La obtención de leche, o uro, es la principal razón de la ganadería mursi. La convivencia de personas y ganado en los poblados, especialmente la presencia en ellos de vacas lactantes, facilita la disponibilidad de leche fresca y también, de forma habitual, de sangre.

Los animales se ordeñan antes de salir a pastar y a última hora de la tarde y para ello se emplea un contenedor de madera denominado bagai. Éstos tienen un cuerpo tubular y un cuello y boca ligeramente más estrechos, el labio recto y la base plana, características que facilitan el consumo y el transporte de la leche fresca (Fig. 9). El primer paso para elaborarlos es cortar un tronco de un árbol común en la llanura central (Cordia gharaf), para posteriormente vaciar el interior de una sección del tronco con un objeto punzante de filo plano o convexo, similar a un cincel. Durante décadas se empleó la punta de una lanza; de hecho, la acción de vaciar uno de estos contenedores se 


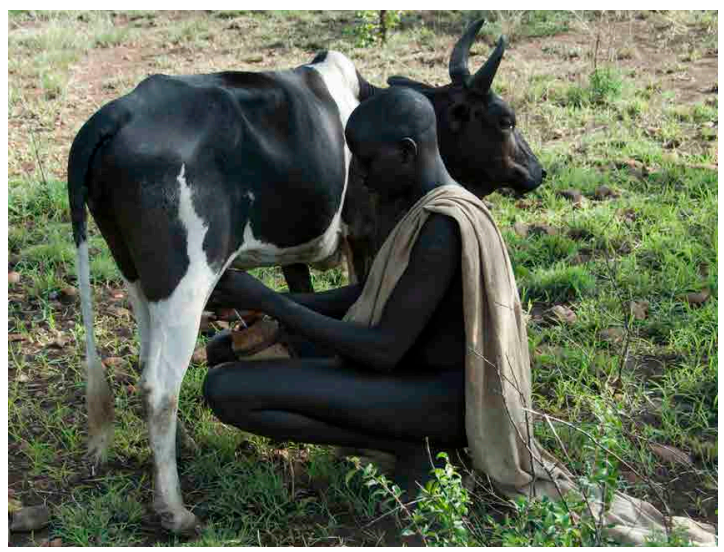

Figura 10. Las hembras del rebaño con una alta producción láctea son considerados animales favoritos, recibiendo todo tipo de cuidados y atenciones.

describe cómo alancear, o kordhi, del mismo modo que se alancea un animal. La persona que ordeña coloca entre las piernas el bagai y dirige el chorro de leche hacia la boca del objeto, consumiéndose habitualmente de forma inmediata el producto obtenido (Fig. 10). La producción media disponible para consumo humano durante el período final de la temporada húmeda ronda los 1.3 litros por vaca y día (Turton 1995). Las mujeres también participan de la obtención y transformación de los productos lácteos, ordeñando y elaborando leche agria o cuajada. Para este último alimento se emplean unas calabazas en las que se deja macerar la leche fresca al sol y, mediante un movimiento de vaivén, obtener el producto final.

En estos mismos asentamientos, y para obtener sangre a partir del ganado, se utiliza un arco y una flecha denominada lawun. Los mursi distinguen entre el arco y la flecha, a los que denominan madre e hijo respectivamente. Tanto el arco como la flecha se realizan con varas de madera (Cordia gharaf), el arco tiene una longitud un metro de longitud y la flecha de medio metro. En un extremo de la flecha se realiza una incisión para colocar una punta de hierro de un centímetro y medio de longitud y sección plana. La acción de extraer sangre del cuello de un animal implica la colaboración de varias personas, siempre del sexo masculino, que ayudan a inmovilizar al animal y exponer su arteria carótida (Fig. 11). Tras disparar la flecha, atravesar la piel y punzar la arteria,

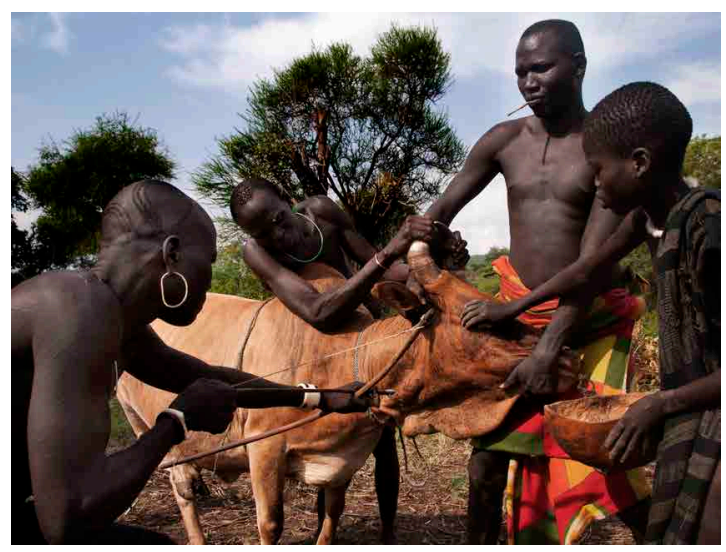

Figura 11. En la acción de obtener sangre, la persona tensa el arco y apunta con la flecha a la arteria a pocos centímetros del animal, mientras que otra persona espera con un recipiente para recoger la sangre cuando comience a brotar.

la sangre se deja brotar por espacio de uno o dos minutos. Una vez obtenida una cantidad de alrededor de medio litro se tapona con barro la herida y se deja marchar al animal con el resto del rebaño. La posibilidad de sangrar a los bovinos y obtener así un alimento alto en proteínas varía según la disponibilidad de pastos, la salud de los animales y la edad de los mismos. En condiciones óptimas, y con animales adultos sanos, es posible sangrarlos cada cinco días, siendo un alimento destinado habitualmente a niños y jóvenes y consumido fresco en los asentamientos.

\section{Una escala del mundo}

Sin olvidar las características individuales de los objetos, son los enlaces matrimoniales los que inician una biografía conjunta de los mismos. Un ôrri a bio es la materialización de una serie de matrimonios. En el desplazamiento de la novia al poblado del novio no solo se traslada la persona con sus conocimientos, capacidades y destrezas, sino que también viajan una serie de objetos, a modo de actores tangibles (Olsen 2013). Así, pieles curtidas para dormir, vasos cerámicos para cocinar, herramientas agrícolas, calabazas para transportar agua y cestas para servir la comida transforman a la adolescente soltera y bajo tutela parental en una agricultora a cargo de sus propias cosechas. Desde hace dos décadas se han incorporado a ese viaje bidones y botellas de plásti- 
co, cacerolas metálicas, prendas de algodón y multitud de otros objetos industriales.

Aunque presenta una relativa simplicidad estructural y tiene un carácter efímero, la casa es el centro y eje vertebrador de esa vida en el asentamiento. Cada vivienda corresponde a una esposa, ya que en la poliginia mursi una mujer no comparte casa con otro cónyuge femenino. Las esposas aseguran el acceso a la tierra cultivable, a un sistema de producción alternativo al ganadero, el agrícola, y su producto principal, el cereal. En caso de no casarse, el hombre está destinado a vivir en los campamentos ganaderos, en un mundo considerado silvestre, sin los múltiples beneficios que provienen de la agricultura, del poblado y de las mujeres. Las casas y el poblado son por tanto el éxito de hombres y mujeres frente al medio, expresan sus habilidades y capacidades para reproducir sus líneas de descendencia y rebaños. Además, mediante ellos garantizan y proyectan su participación pública en la comunidad, un mensaje no verbal claro (Lyons 2009; Lemonnier 2012).

Los ôrri a bio se caracterizan por una marcada división de género, su movilidad espaciotemporal y su autosuficiencia. La cultura material empleada en ellos supone una mímesis continua y poliédrica de estas características. Así, una adolescente experimenta el corte de su labio inferior y la colocación de un primer dilatador de terracota como indicador de su madurez sexual (LaTosky 2006). Posteriormente, ya como esposa, decorará su cuerpo con el plato labial de arcilla cuando sirva la comida en una cesta a su marido, un alimento cocinado en vasos cerámicos. Los platos labiales, los colombinos de la alfarería, las fibras de la cestería o el entramado de ramas de las viviendas son parte de esa capacidad de la mujer mursi para crear cuerpos nuevos a partir de materias locales. Esta fusión continúa de formas, materiales, técnicas, gestos y significados construyen un modelo individual y colectivo de ser mujer.

De forma similar, los hombres son los que tallan la madera para dar forma a diversos objetos, desde un contendedor de leche, al arco y la flecha para sangrar el ganado o a un tablero de juego, incluso cuando el objeto está destinado a las mujeres, como los platos labiales de madera. La acción de tallar la madera y obtener una forma a partir de un cuerpo previo es, en el caso mursi, un ejemplo más de masculinidad. De esta categorización de género no están excluidos los productos ni los lugares. Así, la leche fresca no entrará en contacto con la cerámica y su consumo se realizará en útiles de madera, mientras que el grano se transportará, únicamente, en cestas y pieles. El río Omo, con palmeras y depósitos de arcilla en sus riberas, permiten la cestería y la alfarería, por el contrario, la llanura es un lugar donde tallar múltiples elementos en madera a partir de especies abundantes en ella. Las implicaciones materiales de esta división de género son especialmente visibles durante los desplazamientos, cuando mujeres y hombres, niños y niñas cargan con sus respectivos mundos sobre hombros y cabezas. Objetos y personas forman parte de una misma realidad en ese ejercicio físico y mental de empacar, comprimir y trasladar la totalidad de aquello considerado necesario.

El material empleado, el número de objetos y la tipología y morfología de la cultura material mursi son un aspecto más de la movilidad de las personas. Los movimientos de las personas a lo largo del territorio les aseguran la posibilidad de obtener suficientes recursos como para garantizar su continuidad en el tiempo. El paquete de objetos empleado por cada uno de los grupos familiares de un orri a bio resulta del equilibrio entre sus necesidades y capacidad de transporte. La elaboración de objetos locales a partir de materias abundantes y, por lo tanto, sustituibles y reparables, y no la multifuncionalidad, hace posible esa independencia material. El inventario resultante es un conjunto relativamente reducido y seriado de objetos. Este paquete material ofrece también una escala directa sobre la particular perspectiva mursi del mundo. Así, las capacidades de un contenedor de leche, un vaso para cocinar las gachas o un cesto para servirlas coinciden respectiva, y aproximadamente, con la cantidad de leche obtenida de una vaca por día, la medida necesaria para cocinar para un grupo familiar, también durante un día, o la ración consumida por un adulto en una comida.

Esta escala debe ser recordada, y permanentemente construida, a lo largo de las estaciones y los años, lo que facilita su función vertebradora colectiva. A modo de ejemplo, el círculo mediante el cual la mujer realiza el perímetro de la vivienda lo repetirá en numerosas ocasiones a lo largo de los años y las décadas, y será igual al de sus vecinas. Una mujer casada a los 16 años, y que sobreviva hasta los 60, puede llegar a realizar entre 90 y 120 casas, 
habiendo participado en la construcción de múltiples más. Pero a la construcción de estas casas se deben añadir otro centenar de ocasiones cuando, de adolescentes, han colaborado con la construcción de las casas de sus madres; e incluso antes, cuando de niñas imitan en miniaturas las fuerzas y tensiones necesarias para realizarlas.

\section{Conclusión}

El presente estudio describe, por primera vez, diversos elementos de la cultura material mursi, demostrando la validez de los ôrri a bio como unidad de observación. En ellos se puede apreciar una particular coherencia tecnológica que deriva en la distribución espacial de materiales y personas, la repetición diaria de gestos individuales junto a la flexibilidad de las acciones fruto del comportamiento humano. Además, en las prácticas, las relaciones sociales y las rutinas es posible identificar diversos procesos tecnológicos que permiten elaborar los objetos. En estos asentamientos de la temporada húmeda diversos materiales de procedencia local son transformados en objetos cotidianos. Aunque éstos tienen una apariencia meramente funcional, juegan un papel determinante a la hora de establecer quién pertenece, y cómo lo hace, a la comunidad y, dentro de esta, a un género y a un grupo de edad.

La etnoarqueología permite incorporar a la narrativa científica categorías generadas por las personas con las que se desarrollan los proyectos de estudio. Éstas, a menudo, suponen un desafío a los planteamientos iniciales de los investigadores pero representan quizás la única posibilidad de limitar una perspectiva eurocéntrica. Parece razonable aceptar que cualquier aspiración de comprensión sobre la alteridad se puede beneficiar de la integración en la investigación del mayor número posible de elementos. Además, y en el caso de un estudio sobre los objetos de una población, mediante la incorporación de todo tipo de elementos tangibles se multiplican las posibilidades de observar cambios materiales. Este último factor no es trivial y puede ser determinante si se persigue desarmar la fosilización en el tiempo de las comunidades humanas habitualmente objeto de interés etnoarqueológico.

\section{Agradecimientos}

En primer lugar, agradecer a las comunidades mursi su paciencia y apoyo, con un reconocimiento especial a Olirege Rege y Milisha Olivui, así como a sus familias, por su constante cooperación y amistad. El trabajo no habría sido posible tampoco sin el apoyo de David Turton y de los conservadores del SIP (Valencia), en especial de Bernat Martí y Jaime Vives, así como de una serie de personas que han colaborado en la elaboración de diversas figuras del trabajo, Miguel Crespo, Agustín Díaz y Pilar Mas. Agradecimiento que extendemos a Anna Albiach y a los revisores de la versión previa de este artículo por sus valiosos comentarios y sugerencias.

\section{Bibliografía}

Bassi, M. (2011): Primary identities in the lower Omo valley: migration, cataclysm, conflict and amalgamation, 1750-1910. Journal of Eastern African Studies, 5,1:129-157.

Brittain, M.; Clack, T.; Salazar, J. (2013): Hybridity at the contact zone: Ethnoarchaeological perspectives from the Lower Omo Valley, Ethiopia. Archaeological Review from Cambridge, 28: 133-150.

Bourdieu, P. (1977): Outline of a Theory of Practice. Cambridge University Press, Cambridge.

Clack, T.; Brittain, M. (2011a): Place-making, participative archaeologies and Mursi megaliths: some implications for aspects of pre- and proto-history in the Horn of Africa. Journal of Eastern African Studies, 5 (1), p. 85-107.

Clack, T.; Brittain, M. (2011b): Megaliths, migrations and medicines in Mursiland. Current World Archaeology, 42: 32-39.

Eczet, J.B.; Poissonnier, B. (2012): Décors mobiliers médiévaux et décors corporels actuels: exercice comparatif ethnoarchéologique Shay/Mursi. La Culture Shay d'Ethiopie (Xe-XIVe siècles) (F. X. Fauvelle-Aymar, B. Poissonnier, eds.), Recherches archéologiques et historiques sur une élite païenne, De Boccard/CFEE. 
Epple, S. (ed.) (2014): Creating and Crossing Boundaries in Ethiopia: Dynamics of Social Categorization and Differentiation. African Studies/Afrikanische Studien, 53, LIT Verlag Münster.

Fayers-Kerr, K. N. (2011): The 'Miranda' \& the 'Cultural Archive: From Mun (Mursi) lip-plates, to body painting and back again. Paideuma 58: 245-59.

Foucault, M. (1988): Technologies of the self. Technologies of the Self: A Seminar with Michel Foucault (Martin, L.H. et al. eds.). Tavistock, London: 16-49.

Godelier, M. (1990): Lo ideal y lo material, Pensamiento, economías, sociedades. Taurus, Madrid.

González-Ruibal, A. (2006): The Past is Tomorrow. Towards an Archaeology of the Vanishing Present. Norwegian Archaeological Review, 39 (2), p. 110-125.

González-Ruibal, A.; Hernando, A.; Politis G. (2011): Ontology of the self and material culture: arrowmaking among the Awá hunter-gatherers (Brazil). Journal of Anthropological Archaeology, 30(1): 1-16.

Grillo K. (2014): Pastoralism and Pottery Use: An Ethnoarchaeological Study in Samburu, Kenya. African Archaeological Review 31(2):105-130.

Hodder, I. (1982): Symbols in Action. Cambridge University Press.

Ingold, T. (2007): Materials against materiality. Archaeological Dialogues, 14, no. 1: 1-16.

Jover, F.J. (2013): Las áreas de actividad y las unidades domésticas como unidades de observación de lo social: de las sociedades cazadoras-recolectoras a las agricultoras en el este de la península Ibérica. De la estructura doméstica al espacio social. Lecturas arqueológicas del uso social del espacio (S. Gutiérrez, I. Grau, eds): 13-38.

Latosky, S. (2006): Mursi women's lip-plates as a source of stigma and self-esteem. Perils of face: essays on cultural contact, respect and self-esteem in southern Ethiopia (I. Strecker, J. Lydall, eds.), Lit. Verlag, Berlin: 382-397.

Lemonnier, P. (2012): Mundane Objects, Materiality and Non-verbal Communication. Left Coast Press, Walnut Creek, CA.

Lyon, D. (2009): How I built my house. Ethnoarchaeology, 1(2):137-61.

Olsen, B. (2010): In Defense of Things. Archaeology and the Ontology of Objects, AltaMira Press, New York.

Regi, T. (2008): Mursi duelling, http://mursi.org

Robb, J. (1998): The Archaeology of Symbols. Annual Review of Anthropology, 27: 329-346.

Schlanger, N. (2012, 23 Abril): Material Culture': The Concept and its Use in Historical Perspective [Video]. Recuperado de http:// www.youtube.com/watch?v=WD8mopXzfmQ

Turton, D. (1973): The social organisation of the Mursi, a pastoral tribe of the lower Omo Valley, southwestern Ethiopia. London School of Economics, University of London.

Turton, D. (1979): A Journey Made Them: Territorial Segmentation and Ethnic Identity among the Mursi. Segmentary Lineage Systems Reconsidered (L. Holy, ed.), Queen's University Papers in Social Anthropology 4, Queen's University, Belfast: 119-43.

Turton, D. (1995): Pastoral Livelihoods in Danger: Cattle Disease, Drought and Wildlife Conservation in Mursiland, Southwestern Ethiopia. Oxfam Research Papers No. 12, Oxfam.

Turton, D. (2002): The same only different: war and duelling as boundary marking rituals in Mursiland, southwestern Ethiopia. War and Games (T. Cornell, ed.) Boydel \& Brewer, Woodbridge, Suffolk: 17192.

Turton, D. (2011): Wilderness, Wasteland or Home? Three ways of imagining the Lower Omo Valley. Journal of Eastern African Studies 5(1): 158-176. 